\title{
INTRODUCCIÓN: EN TORNO AL ORIGEN DEL PRIMER ALIENISMO
}

\author{
Juan Carlos Stagnaro (Coordinador) \\ Profesor Regular Titular, Departamento de Psiquiatría y Salud Mental y Docente e Investigador del \\ Instituto de Historia de la Medicina, Facultad de Medicina, Universidad de Buenos Aires \\ jcstagnaro@gmail.com
}

Recibido: 12 de abril de 2015; Aceptado: 10 de mayo de 2015.

Cómo citar este artículo/Citation: Stagnaro, Juan Carlos (2015), "Introducción: En torno al origen del primer alienismo", Asclepio, 67 (2): p104. doi: http://dx.doi.org/10.3989/asclepio.2015.22

\section{INTRODUCTION: ABOUT THE ORIGIN OF THE FIRST ALIENISM}

Existe toda una tradición historiográfica que identifica el nacimiento de la psiquiatría con la figura de Philippe Pinel (1745-1826), cuyo nombre quedó registrado como epónimo del origen de la especialidad.

Aunque investigaciones posteriores matizaron ese lugar mítico del clínico francés, en el imaginario colectivo se le sigue atribuyendo todo el crédito del origen de la medicina mental, tanto en lo referente a la famosa "liberación de los locos de sus cadenas" como, utilizando la expresión foucaultiana, del «nacimiento de la clínica (psiquiatría)», sin tener suficientemente en cuenta que dicho momento fundacional de la psiquiatría respondió a un proceso complejo que se desarrolló de manera más o menos simultánea en diversos lugares de Europa, en un marco intelectual (fi- lantrópico e ilustrado) que propició una nueva forma de entender la locura y de actuar sobre el loco.

En efecto, se acepta situar el comienzo de una nueva especialidad médica - primero conocida como alienismo y luego como psiquiatría - orientada al estudio y tratamiento de la locura, hacia 1800, en París, en la época en la que Pinel, profesor de Nosographie de la Facultad de Medicina de Paris, fue designado director del hospicio de Bicêtre por el Comité de Salud Pública de la Revolución Francesa.

Sin embargo, como lo señala el historiador de la psiquiatría Jacques Postel (1981), la atribución exclusiva a Pinel de ese fenómeno histórico tiene algo de excesivo. Efectivamente, corría a la sazón en Europa un viento de reforma social que también tuvo su ex- 
presión en el pensamiento médico sobre la locura; y esa corriente de opinión se plasmó antes, durante y después de la producción pineliana, en las obras y acciones institucionales de médicos de diversos lugares del viejo continente: Johann Christian Reil en Alemania, Vincenzo Chiaruggi en Florencia, Joseph Daquin en Chambery, William Tuke en "El Retiro" de York, J. Theodor Held en Praga y, tiempo después, F. Sabler en Moscú, entre los más importantes. Todos ellos hicieron aportes a una nueva clínica de la locura, denunciaron la situación de los enfermos mentales y propusieron cambios y, en algunos casos, lograron plasmar experiencias en las que aplicaron los principios del Movimiento Filantrópico en coincidencia con las ideas de enciclopedistas y librepensadores del siglo XVIII que proponían una suerte de secularización de la caridad.

Sin embargo, el mérito de ese movimiento quedó ligado al médico francés: durante más de un siglo, gracias a los escritos laudatorios de su hijo Scipión y de su sobrino bisnieto René Semelaigne (1888), se difundió y se mantuvo el mito pineliano.

Aunque debe aceptarse también que la atribución a Pinel del acontecimiento que estudiamos también se debe, en gran medida, al lugar central que ocupaba Francia dentro de la cultura europea en los años de la Revolución Francesa y a que él logró sintetizar, como ningún otro de sus contemporáneos, los presupuestos de una nueva forma de concebir la locura y su tratamiento.

\section{LA CRÍTICA DEL MOVIMIENTO FILANTRÓPICO A LOS HOSPITALES GENERALES: COLOMBIER Y DOUBLET}

A lo largo de los siglos XVII y XVIII en Europa, los locos o bien eran cuidados por sus familias en condiciones muy precarias, tratados por la medicina con los métodos clásicos heredados de la tradición hipocrático-galénica combinada con los más diversos y iatrogénicos recursos, o vagaban por campos y ciudades mendigando la ayuda pública o bien eran recluidos, junto a prostitutas, ancianos indigentes, niños abandonados, delincuentes comunes y vagabundos en condiciones miserables en los Hospitales Generales verdaderas prisiones con un alto índice de mortalidad y no pocas fugas - que habían creado las monarquías para sacar de circulación a los considerados "perturbadores" del orden social.

Los enfermos mentales formaban una suerte de casta estigmatizada como indigna de la condición humana. Algunas de las instituciones que los albergaban eran visitadas por los curiosos a cambio de una propina para sus cuidadores.

En la Francia del Ancien Régime el encierro de los locos en los Hospitales Generales estaba legalmente pautado. Las familias podían solicitar al soberano el encierro de sus deudos delirantes, furiosos, libertinos, pródigos, escandalosos, etc., por medio de la emisión de una Orden del Rey (Lettre de cachet), procedimiento expeditivo que conducía a la internación del señalado como enfermo. En el caso de los sujetos errantes, generalmente indigentes y sin familia, era la autoridad policial, en Paris, o los intendentes en las provincias, quienes podían acudir a la autoridad real con el mismo propósito.

A comienzos del reinado de Luis XVI había irrumpido en la escena política un movimiento que, inspirado en el ideario de Jean-Jacques Rousseau, se definirá por "una repugnancia innata" a ver sufrir al semejante. Era el denominado movimiento filantrópico que, en el marco de una creciente conflictiva política con la monarquía, al calor de las ideas divulgadas por los enciclopedistas y sinérgico con las reivindicaciones del Tercer Estado, desveló, entre otras denuncias, las miserables condiciones en las que se encontraban los recluidos en los Hospitales Generales del reino. En 1776, cuando Jacques Necker sucedió a Turgot en el cargo de Primer Ministro, el problema de la asistencia estaba a la orden del día: para los filántropos la miseria era producto de las condiciones de vida de la sociedad y, por lo tanto, era ella quien debía reparar el mal que causaba, y el Estado, responsable de la política social, no podía abandonar la provisión de asistencia solamente a la caridad.

En ese tormentoso contexto social pre-revolucionario el ministro Necker designó, en 1780, a Jean Colombier (1736-1789) en el cargo de Inspector General de Hospitales civiles y Prisiones del reino.

Poco antes de su caída, Luis XVI, bajo la presión de severas denuncias presentadas por los simpatizantes del movimiento filantrópico, encomendó a Colombier y a su adjunto, François Doublet (1751-1795), la redacción de un informe sobre el estado de los locos internados en los Hospitales Generales del reino. Como resultado de una gira por todas las instituciones de Francia destinadas a alojar alienados, ambos médicos presentaron, en 1785, su famoso texto: Instruction sur la manière de gouverner les insensés, et de travailler à leur guerison dans les asyles qui leur sont destinées, que sentó las bases esenciales del modelo del asilo terapéutico que se pondrían en práctica a principios del siglo XIX. 
Un elocuente pasaje de la Instruction... da una idea de la miserable existencia en la que se encontraban las personas recluidas en los Hospitales Generales: " millares de insanos son encerrados en las prisiones sin que nadie piense en el menor de los remedios. El semiinsano que se confunde con el insano perdido; el furibundo con el loco tranquilo: a unos se los encadena, a otros se les deja en libertad en su cárcel; en resumidas cuentas, a no ser que la naturaleza acuda en su auxilio y los cure, el término de sus males es el de sus días, y desgraciadamente hasta entonces, la enfermedad no hace sino aumentar en lugar de disminuir" (Colombier y Doublet, 1785, p. 4).

Pero las intenciones de reforma se vieron interrumpidas por la Revolución de 1789.

El informe de Colombier y Doublet, que fue ampliamente difundido por todo el reino, conserva una gran importancia histórica por ser el primer documento oficial de carácter reformador que, poco antes de producirse la Revolución, se ocupó de la situación de los enfermos mentales en Francia, indicando los criterios que la medicina de fines del siglo XVIII debía observar en relación a ellos. A pesar de su breve vigencia, debido a la caída de la monarquía, la Instruction tuvo una gran repercusión porque su contenido fue retomado por el Comité de Mendicidad de la Asamblea Constituyente.

Los miembros de ese estamento, sobrecogidos ante los miles de personas que habían alojado los Hospitales Generales entre 1768 y 1789, pusieron en práctica las recomendaciones de Colombier y Doublet, designando a Pinel en el hospicio de Bicêtre con la misión de reformarlo de manera acorde con las exigencias de los nuevos Derechos del Hombre instaurados por la Revolución (Weiner, 2002, pp. 109-110). Sin embargo, antes de estudiar la obra de Pinel es necesario, a fin de tener una visión de conjunto de lo señalado antes, pasar revista a lo que estaba ocurriendo en otros lugares de Europa.

\section{GRAN BRETAÑA: WILLIAM BATTIE Y JOHN MONRO, JOHN HASLAM, ALEXANDER CRICHTON}

Tres décadas antes de la elaboración de la Instruction de Colombier y Doublet, tuvo lugar una áspera polémica pública entre los médicos londinenses WiIliam Battie y John Monro. A poco de comenzar su tarea como director del Saint Luke's Hospital for Lunatics de Londres ${ }^{1}$, el doctor William Battie (1703-1776) inició su prédica en defensa de un tratamiento más humano de los enfermos mentales que plasmó en su Treatise on Madness (1758), que fue la primera mo- nografía médica inglesa dedicada al tema. Adscribiendo a las ideas del Iluminismo, Battie abogó por una visión optimista sobre el modo de tratar los trastornos mentales, oponiéndose a la utilización indiscriminada de laxantes, eméticos y sangrías profusas e ineficaces habitualmente practicadas en el Hospital de Bethlem (popularmente llamado Bedlam), a la sazón la institución para alienados más antigua y conocida de Londres. En su opúsculo, además de criticar los métodos utilizados en esa institución, a la que tachaba de reducto de la barbarie y la coerción, Battie denunció la práctica de permitir las visitas de los vecinos de la ciudad, quienes por unas monedas podían espiar las celdas en las que estaban recluidos los enfermos.

Se dice que durante su carrera, Battie había examinado a más de mil enfermos mentales por lo que no cabe duda de que debió acumular una gran experiencia clínica y de que su célebre libro constituyó un interesante ejemplo de teorización médica en torno a la explicación del desvío y la intensidad de las pasiones como causa de la locura. Battie también abogaba en su obra por un trato más humano de los pacientes, proporcionándoles mejores condiciones de habitación en celdas aireadas, buena alimentación y distracciones mediante la visita de sus familiares y amigos.

Ese libro inglés marcó un hito decisivo en el enfoque médico de la enfermedad mental; y su promoción del optimismo terapéutico, a través del compromiso con el paciente, en lugar de la restricción u otras maniobras físicas, prefiguró el tratamiento moral que pusieron en práctica los Tuke en "El Retiro" de York, a finales del siglo XVIII, y que aplicó, inicialmente, Daquin en Chambèry $y$, finalmente, perfeccionaron Pinel y Esquirol (1772-1840) en los hospitales de París.

El libro de Battie suscitó la inmediata y airada réplica del doctor John Monro (1715-1791), director, como su padre, del Bedlam. Monro interpretó el escrito de Battie como un ataque a la memoria y buen nombre de su progenitor, y de él mismo y respondió inmediatamente con un texto intitulado Remarks on Dr. Battie's Treatise on madness (1758).

Pero, lo más importante, aparte del valor anecdótico de esa controversia, es que el cruce entre ambos médicos constituyó el primer debate acerca de la teoría de la locura y de su tratamiento en suelo inglés (Morris, 2008).

John Haslam (1764-1844), médico londinense, muy distinguido en su medio en razón de sus trabajos científicos y sus notas periodísticas, adquirió una gran reputación por su moderación en el tratamiento 
de los enfermos mentales. En 1795 se hizo cargo de la botica del hospital de Bethlem, introduciendo los más modernos criterios científicos en la farmacopea del establecimiento. Esa tarea le dio la oportunidad de observar, cotidianamente, el comportamiento de los pacientes internados en su libro Observations on Insanity With Practical Remarks on the Disease and an Account of the Morbid Appearances on Dissection (1798), en el que incluyó las descripciones detalladas de las enfermedades más habituales que sufrían, así como el resultado de las disecciones cerebrales de aquellos que morían.

Pero la trayectoria profesional de Haslam se vio marcada por un acontecimiento conflictivo. En mayo de 1814, Edward Wakefield (1774-1854) un reformador y filántropo, miembro del Parlamento británico, realizó una visita de inspección al hospital de Betlhem y descubrió en una de sus celdas a un ciudadano norteamericano, de apellido Norris, encadenado por indicación de Haslam, quien lo juzgaba peligroso a partir de un altercado que había tenido con él, a un dispositivo de hierro que lo mantenía permanentemente desde hacía una decena de años en atroces condiciones.

Alertados por Wakefield varios miembros del Parlamento visitaron al paciente y estuvieron de acuerdo en que era un hombre racional y tranquilo. Aunque liberado de sus ataduras el norteamericano, ya agotado físicamente por los malos tratos recibidos murió en el hospital pocos meses después. El caso de Norris produjo una gran conmoción en la opinión pública y contribuyó al movimiento de reforma de los asilos del Reino Unido, plasmado en la legislación de 1828 que regularizó el funcionamiento de las instituciones para los enfermos mentales. A la base de estas iniciativas estuvieron, precisamente, Edward Wakefield, William Hone, escritor político y editor, y James Bevans, arquitecto, quienes se preocuparon por la condición y el mal trato de los pacientes en los asilos y formaron un comité destinado a visitar a ese tipo de instituciones de todo el país y a confeccionar informes sobre los hallazgos (Andrews et al, 1997, pp. 415-435).

En 1798, otro médico escocés, Alexander Crichton (1763-1856), publicó An Inquiry into the Nature and Origin of Mental Derangement (1798) que fue traducido al holandés, alemán y, en parte, al francés. La obra de Crichton, constituyó un antecedente singular para el nacimiento de la psiquiatría y ejerció una gran influencia en los pioneros franceses de la especialidad, como Pinel y Esquirol (Berrios, 2006; Charland, 2008).

\section{LA EXPERIENCIA DE CHAMBÉRY Y EL PENSAMIENTO DE JOSEPH DAQUIN}

En Chambéry, capital del antiguo ducado de Saboya (Reino de Piamonte-Cerdeña), Joseph Daquin (1732-1815) publicó, en 1791, la primera edición de su libro intitulado Philosophie de la folie, que tuvo una segunda edición, en 1792, en Paris, idéntica a la primera en su contenido, pero con una modificación en el título: Philosophie de la folie ou essai philosophique sur le traitement des persones attaqués de folie y, tres años más tarde, en 1804, una tercera edición "revisada, aumentada y apoyada en un gran número de observaciones".

En su obra, Daquin sostiene que la enfermedad mental debe ser tratada por medio del tratamiento moral, término que retoma Pinel, y comunica sus investigaciones sobre la influencia de la luna en la génesis de la locura.

Daquin falleció discretamente, sin que sus opiniones y propuestas terapéuticas sobre la locura alcanzaran una difusión particular. La repercusión de la obra de Pinel opacó sus anticipaciones, sobretodo porque el gran maestro parisino, así como su discípulo Esquirol, nunca reconocieron la contribución pionera de Daquin.

Si bien, como lo afirma el historiador Claude Quétel (1987), es ocioso, y hasta incorrecto históricamente, discutir la preeminencia de Pinel en la invención completa del tratamiento moral, también es cierto que él no menciona la contribución de Daquin, la cual, por muy incipiente que fuera, no carecía de originalidad. Como también es cierto que la Philosophie de la foliefue el primer libro consagrado íntegramente al estudio de la locura que se haya publicado en Francia.

\section{VINCENZO CHIARUGI EN FLORENCIA}

El Duque de la Toscana Pedro Leopoldo, promulgó, a finales de 1774, en el marco de profundas reformas sociales y administrativas que caracterizaron su gobierno, la primera ley sobre los enfermos mentales de Europa (Legge sui pazzi), que codificaba la relación de la sociedad con esas personas y el modo de su hospitalización. Según ese decreto Real nadie, ni siquiera pagando, podía ser admitido en el Manicomio para su custodia o cura, sin previa certificación de la enfermedad firmada por médico y sin una licencia soberana para internarlo.

La nueva administración había realizado cambios y reformas en las instituciones florentinas destinadas a 
recibir enfermos mentales y, en ese proyecto incluyó el Regolamento dei Regi Spedali di Santa Maria Nuova e di Bonifacio, inspirado por Vincenzo Chiarugi (17591820) y puesto en vigencia en 1789 , que constituyó la prolongación de las legge sui pazzi en el plano sanitario.

El Regolamento concebido por Chiarugi representó el primer texto en el que el reformismo del siglo XVIII se plasmó en una institución de ese tipo. En la práctica, las directivas promulgadas fijaban como una prioridad, con un fin tanto ético como terapéutico, el respeto del enfermo bajo los aspectos físico y psicológico; lo cual significó la abolición de los malos tratos con pretensión curativa. Cesaron así la inmovilización con cadenas - aunque se la mantuvo para los más agitados o con riesgo de suicidio empleando solo cintas de tela y muñequeras de cuero adecuadamente puestas para evitar heridas y dolor-, las sangrías profusas y/o repetidas y las inmersiones en agua fría; $y$, sobre todo, se dispuso la presencia constante de un médico en el hospital y la supervisión del personal auxiliar. En su conjunto quedó fuertemente marcada la diferencia entre la finalidad sanitaria de la nueva institución y el objetivo meramente cautelar y segregatorio del antiguo asilo (Stagnaro, 2014).

En la misma época en la República de Luca se inauguraba, en 1773, el hospital de San Luca della Misericordia, y poco después el de Fregonaia, con el mismo propósito de mejorar la atención de los alienados. Como afirma Georges Lanteri-Laura (1998), estos acontecimientos muestran que la tesis de Michel Foucault ubicando al "gran encierro" como el origen de la psiquiatría moderna debe ser revisada en ese sentido, y no puede tener la pretensión de dar cuenta de todo lo que se pensaba y pasaba en ese momento en la Europa de las Luces.

En 1793 se publicó en la imprenta de Luigi Carlieri el primer Tomo del libro de Chiarugi Della Pazzia in genere, e in specie. Trattato médico-analitico con una centuria di osservazioni, al que le siguió, en 1794, un segundo volumen conteniendo los Tomos II y III, que fueron traducidos al alemán dos años después (1793-1794 [2014]).

Ese texto, que dejó marcado indeleblemente el nombre de Chiarugi en la historia de la psiquiatría, fue profundamente modificado por su autor para una segunda edición aparecida en Florencia en 1808. Pero, para esa fecha, ya estaba circulando la primera edición del Traité médico-philosophique sur l'aliénation mentale ou La manie de Pinel (1800), y muy pronto a salir la segunda edición de esa obra (Pinel, 1809) que se había difundido por toda Europa.
Si bien el francés formuló una teorización del fenómeno psicopatológico más avanzada, el toscano llevó adelante una transformación hospitalaria más audaz y precoz, pero ambos fueron participes del movimiento médico europeo que revolucionó la recepción y el tratamiento de los enfermos mentales en la segunda mitad del siglo XVIII y los albores del siglo XIX (Stagnaro, 2014)

\section{ALEMANIA: LAS RAPSODIAS DE REIL}

Finalmente, en Alemania, los conceptos y argumentaciones incluidos en el famoso trabajo de Johann Christian Reil (1759-1813) de 1808, en el que defiende la creación de una disciplina médica independiente denominada Psiquiatría (Reil, 1808), se basaron en gran medida en sus Rhapsidien uber die Anwendung der psychischen Kurmethode auf Geisteszeruttungen (1803) Muchos de esos argumentos continúan siendo claves para definir la especialidad en el presente. Reil reflexionó sobre sus aspectos principales e incluso planteó los derechos de los enfermos mentales, denunció los efectos del estigma que portan, defendió la creación de hospitales humanizados para ellos y enfatizó la responsabilidad del gobierno y la sociedad toda ante sus ciudadanos aquejados de esos trastornos. Y, fundamentalmente, describió a la psicoterapia como un tratamiento esencial, tanto para las enfermedades mentales como para las somáticas, equivalente a los tratamientos farmacológicos y a la cirugía (Garrabé, 2008).

\section{LA INTERPRETACIÓN DE LA OBRA DE PINEL}

El libro de Gladys Swain Le Sujet de la folie (1977), y el publicado junto a Marcel Gauchet La pratique de l'esprit humain, l'institution asilaire et la révolution démocratique (1980), retoman la investigación sobre los orígenes de la psiquiatría, dedicándose a mostrar que los comienzos del saber psiquiátrico, alrededor de 1800, representan una fecha decisiva para las ciencias del hombre. Los autores mencionados inauguraron una nueva interpretación sobre el tema de la sinrazón en el Occidente moderno, que $\mathrm{M}$. Foucault había descrito como una vasta reducción al silencio que culminaba con la pretensión de la razón de proveerse una ciencia objetiva para el alienado. La reconstitución que aportó Swain, del momento fundacional de la clínica psiquiátrica en sus términos exactos, nos ofrece una imagen profundamente diferente: allí donde la tradición veía un loco encerrado en la soledad de su delirio o la ceguera de su furia, los médicos descubrieron bruscamente un ser que no 
está cerrado a los otros ni a lo que le acontece. $\mathrm{Ni}$ encierro en sí ni ausencia de sí en el alienado, sino una desgarrante presencia que se interroga sobre el sentido de su trastorno y una constante preocupación por el otro. De allí la posibilidad de acceder a un intercambio terapéutico con ese sujeto que sabe algo de su alienación y se defiende contra ella. En otras palabras, Swain y Gauchet pusieron el acento sobre el concepto fundamental de la empresa de $\mathrm{Pi}$ nel: cualquiera sea el grado de locura del alienado, este último no está nunca completamente ajeno a sí mismo; el paciente no adhiere jamás totalmente a su propia alienación. Es precisamente esa distancia entre el loco y su propia locura lo que hace posible en los hospicios de Bicêtre y La Salpêtriere emprender el "tratamiento moral"; ya que se podrá establecer con esa parte sensata del insensato el diálogo terapéutico. Mientras Michel Foucault en su Histoire de la folie à l'âge classique (1961) hace de Pinel un heredero directo de toda una tradición, Swain y Gauchet subrayan, por el contrario, el valor de ruptura teórica de su propuesta. Si bien es cierto que retirar a los locos del Hospital General para alojarlos en asilos específicos dio testimonio de una voluntad de exclusión, también debe reconocerse que ese aislamiento en las instituciones monovalentes constituyó a la locura como objeto teórico a parte entera. $Y$ es, entonces, en ese momento, en el que el loco devino el objeto de una verdadera investigación científica que se impuso la presencia de la parte inalienable de su subjetividad.

\section{La sanción legal del nuevo orden de la locura}

La obra clínica y terapéutica de Pinel se completó con una legislación que sancionó, diez años después de su muerte, el marco jurídico de la internación psiquiátrica. Dos de los principales alumnos de Pinel, Esquirol y Ferrus (1784 - 1861) fueron los promotores de la famosa ley promulgada el 30 de junio de 1838 bajo la monarquía de Julio que reglamentó por más de 150 años en Francia (recién fue modificada en 1990) la hospitalización de los enfermos mentales en los establecimientos privados y públicos, su protección y la de sus bienes. Inspirados en los ideales filantrópicos y humanistas del siglo XVIII se creó así una doctrina de la psiquiatría en Francia (que sirvió como modelo para numerosos países) con las siguientes características:

a) Creó condiciones decentes de recepción y de tratamiento para los enfermos mentales, imponiendo a cada Departamento geográfico de Francia la organización y la carga financiera de esas estructuras hospitalarias: los asilos de alienados. b) Dictaminó una serie de medidas de protección social contra los riesgos de la peligrosidad de los enfermos mentales sobre la población general.

c) Reglamentó de manera precisa las circunstancias en las cuales un ciudadano podía ser encerrado en un asilo de alienados contra su voluntad, y las medidas de protección legal para la persona, tanto en lo relativo a sus bienes como a su libertad individual.

La ley de 1838 se propuso poner fin, definitivamente, a las prácticas brutales de tratamiento de la enfermedad mental que prevalecían hasta entonces. Podría decirse, y a justo título, que dicho instrumento legal respondía, simultáneamente, más allá del espíritu humanista de sus mentores, a una lógica que puede calificarse de represiva; fundamentalmente creada para proteger al cuerpo social del efecto desestabilizador del trastorno social provocado por la locura. Pero deberá reconocerse también que entre los beneficios novedosos que otorgó se cuenta la legitimación de la medicina para el tratamiento de la enfermedad mental.

En efecto, el reconocimiento legal del médico alienista y de la disciplina psiquiátrica revistió una importancia capital: la medicina limitada por una deontología profesional debió reconocer en el enfermo mental el derecho a la calidad de los cuidados que debía recibir y el respeto a su dignidad como persona.

\section{EL PSIQUIATRA Y LA INSTITUCIÓN}

La institución psiquiátrica formó así, desde su origen, parte indisoluble de un dispositivo terapéutico pensado para modificar la conducta desviada designada por Pinel y sus contemporáneos como alineación mental. Su disposición edilicia, su estructura organizativa (médico jefe, enfermeros guardianes), la admisión, salida y circulación de sus internos ("secuestro" y encierro obligado hasta el alta decidida por la autoridad médica), sus actividades internas (clinoterapia, tratamientos biológicos, tratamiento moral, laborterapia, recreación y visitas dosificadas de los allegados), no surgió del capricho autoritario de sus creadores sino de una serie de maniobras terapéuticas enderezadas a modificar, a restablecer el estado previo de lucidez y razonamiento claro de los considerados enfermos de alienación mental.

Fue así como, en un entrelazamiento inextricable, la especialidad nació entre los muros del asilo del Ancien Régime, el cual se transformó, a su vez, en función de una teoría de la locura que lo utilizó como herramienta, en el hospital psiquiátrico. 
A este respecto, en el prólogo a la obra Miserables y locos. Medicina mental y orden social en la España del siglo XIX, de Fernando Alvarez Uría, Robert Castel señala que "El nacimiento de la psiquiatría en el siglo XIX fue, sin duda alguna, una innovación considerable: la creación de una nueva institución -el manicomio-, de una legislación especial nueva, de un cuerpo de nuevos especialistas - los médicos psiquiatras-, de un estatuto del loco convertido ahora en enfermo, etc. En estas decisivas transformaciones, los historiadores de la medicina suelen ver de buen grado una «revolución» y el comienzo de una era radicalmente nueva. La importancia del cambio no debe, sin embargo, ocultar que tal cambio reconduce determinados rasgos fundamentales de dicha situación y que estas permanencias tienen, por lo menos, tanto peso como las innovaciones, en la comprensión del significado del fenómeno. Así, la necesidad del "aislamiento terapéutico", en cuyo nombre todo el nuevo sistema se pone en marcha, reconduce la segregación anterior de los alienados en los hospitales generales y en las prisiones; el estatuto de minoría legal del enfermo mental es homólogo al estatuto del condenado, tal como aparece en el código penal, puesto que tanto uno como el otro se ven privados de sus derechos; la tutela médica de la razón sobre la locura se ejerce con la misma buena conciencia que la tutela jurídica del juez sobre el delincuente, etc. La primera 'revolución psiquiátrica', la que se produjo en el momento de Pinel [...] supone el paso de una lógica directamente represiva (salvaguarda del orden público - arresto - juicio - secuestro) a una lógica médico-humanista (interés del enfermo - aislamiento - ingreso en un establecimiento especial - tratamiento médico). Sin embargo, "¿ies lo mismo una cosa que otra?» — se interroga Castel- «en absoluto» - afirma - "se trata de un desplazamiento esencial que permite la emergencia de ese conjunto considerable de prácticas nuevas que van a desarrollarse bajo el label de la medicalización» (Castel, 1983, pp. 9-10).

\section{CONCLUSIÓN}

Es necesario analizar más de cerca, con instrumentos historiográficos más adecuados, los conceptos que, cargados de un fardo ideológico frecuentemente no explicitado, tienden a presentar el nacimiento de la psiquiatría exclusivamente como una maniobra de apropiación de la locura en el campo de la medicina con fines de control social. Un correcto estudio de los hechos puede permitirnos descubrir que el surgimiento de la especialidad, aunque no se desembarazó de ciertas dimensiones heredadas inspiradas en el miedo a la locura y su marginación, que estaban y están inscritos en nuestra cultura, constituyó una transformación positiva de la forma en que la sociedad occidental comenzó a ocuparse de la locura en las últimas décadas del siglo XVIII y primeras del XIX.

El Dossier monográfico que se propone en este número de Asclepio, pretende - sin agotar la lista de los protagonistas - a través de determinados estudios de caso: Joseph Daquin en el Piamonte, Alexander Crichton en Gran Bretaña, Vincenzo Chiarugi en Italia y Johann Christian Reil en Alemania, analizar la obra institucional y los conceptos centrales de esos autores, los cuales desempeñaron un papel central en el proceso que se pretende estudiar.

La tesis que este conjunto de investigaciones pretende sustentar es que las nociones que dieron lugar a la medicalización de la locura, al tratamiento moral y al inicio del movimiento alienista, se fue delineando desde mediados del siglo XVIII y que la psiquiatría surgió de nuevos conceptos y prácticas diversas y convergentes desarrolladas en diversos países europeos cristalizando, finalmente, en la obra de Pinel con la segunda edición de su Traité en 1809: punto de llegada y no tanto de partida del pensamiento médico moderno sobre las enfermedades mentales.

Este Dossier se originó en un simposio intitulado: "The birth of psychiatry: an European movement» celebrado en el marco del XVI Congreso Mundial de Psiquiatría realizado en Madrid en septiembre de 2014. El éxito de convocatoria y las valoraciones de los asistentes nos han animado a escribir las ponencias allí presentadas. Los autores, especialistas en historia de la psiquiatría, forman parte de la Red Iberoamericana de Historia de la Psiquiatría y llevan años trabajando y debatiendo conjuntamente sobre las relaciones entre historia y clínica en el ámbito de la psiquiatría.

a Hunter Richard; Macalpine, Ida (1962), “Introduction”. En Hunter Rilliam A. Treatise on Madness. London, Dawsons of Pall Mall., y está tomada de Berrios (1993, p. 32). 


\section{BIBLIOGRAFÍA}

Andrew, Jonathan; Asa Briggs; Roy Porter; Penny Tucker; Keir Walddington (1997), The History of Bethlem. London and New Tork, Routledge.

Battie, William (1758), Treatise on Madness. London, Whiston and White [Versión castellana: Battie, William. Tratado sobre la locura. En: AA.VV. Los prolegómenos del tratamiento moral. Colección Clásicos de la Psiquiatría. Buenos Aires, Polemos, 2013].

Berrios, German E. (1993), "Los psiquiatras británicos y su formación", Revista de la Asociación Española de Neuropsiquiatría, 13, (Suplemento II), pp. 32-36.

Berrios, German E. (2006), "Alexander Crichton and general mind", History of Psychiatry, 17, pp. 469-498.

Castel, Robert (1983), "Prólogo". En: Alvarez Uría, Fernando, Miserables y locos. Medicina mental y orden social en la España del siglo XIX, Tusquets, Barcelona, pp. 7-13.

Colombier, Jean y François Doublet (1785), Instruction sur la manière de gouverner les insensés, et de travailler à leur guerison dans les asyles qui leur sont destinées. Paris, I'Imprimerie Royal [Versión castellana: Colombier Jean; Doublet, François. Instrucción sobre la manera de gobernar a los insensatos y trabajar para su curación en los asilos que les son destinados. En: AA.VV. Los prolegómenos del tratamiento moral. Colección Clásicos de la Psiquiatría. Buenos Aires, Polemos, 2013].

Charland, Louis C. (2008), "Alexander Crichton on the psychopathology of the passions", History of Psychiatry, 19(3), pp. 275-6.

Chiarugi, Vincenzo (1793-1794), Della Pazzia in genere, e in specie. Trattato médico-analitico con una centuria di osservazioni. Firenze, Carlieri [Versión castellana: Chiarugi Vincenzo, La Locura, sus géneros y especies. Tratado médico-analítico con cien observaciones. 1a edición en castellano. Colección Clásicos de la Psiquiatría, Buenos Aires, Polemos, 2014].

Crichton Alexander (1798), An Inquiry into the Nature and Origin of Mental Derangement. London, Candell Jr. and Davies [Versión castellana: Crichton, Alexander. Investigación sobre la naturaleza y el origen del trastorno mental. En AA. VV. Los prolegómenos del tratamiento moral. Colección Clásicos de la Psiquiatría, Buenos Aires, Polemos, 2013].

Daquin Joseph (1791), La philosophie de la folie, Gorrin père et fils, Chambery, Réed. Paris, Insania [Versiones castellanas: Daquin, Joseph. Filosofía de la locura. Madrid, Asociación Española de Neuropsiquiatría (AEN), 2000 y en AA. VV. Los prolegómenos del tratamiento moral. Colección Clásicos de la Psiquiatría. Buenos Aires, Polemos, 2013].

Foucault, Michel (1961), Folie et déraison. Histoire de la folie à l'âge classique. Paris, Plon.
Garrabé, Jean (2008), “À propos de 'Rhapsodies sur l'emploi d'une méthode de cure psychique dans les dérangements de l'esprit', traduit en français para Marc Géraud", L'Évolution Psychiatrique, 73, pp.418-427.

Gauchet, Marcel y Gladys Swayn (1980), La pratique de l'esprit humain. L'institution asilaire et la révolution démocratique. Paris, Gallimard.

Haslam, John (1798), Observations on Insanity With Practical Remarks on the Disease and an Account of the Morbid Appearances on Dissection. London, Rivington.

Lantéri-Laura, Georges (1998), Essai sur les paradigmes de la psychiatrie moderne. Paris, Éditions du temps.

Monro, John (1758), Remarks on Dr. Battie's Treatise on madness. London, Clarke [Versión castellana: Monro, John. Comentarios acerca del Tratado sobre la locura del Dr. Battie. En: AA. VV. Los prolegómenos del tratamiento moral. Colección Clásicos de la Psiquiatría. Buenos Aires, Polemos, 2013].

Morris, Andrew (2008), "William Battie's Treatise on Madness (1758) and John Monro's Remarks on Dr Battie's Treatise (1758) - 250 years ago", British Journal of Psychiatry, 192 (4), p. 257.

Pinel, Philippe (1800), Traité médico philosophique sur l'alienation mentale ou la manie. Paris, Caille et Ravier [Versión castellana: Pinel, Philippe. Tratado médico-filosófico de la enagenación mental o manía. Madrid, Ediciones Nieva, 1988].

Pinel, Philippe (1809), Traité médico philosophique sur l'alienation mentale, 2ème éd. Paris. Brosson [Versión castellana: Pinel, Philippe. Tratado médico-filosófico sobre la alienación mental. En: AA. VV. El nacimiento de la psiquiatría. Colección Clásicos de la Psiquiatría. Buenos Aires, Polemos, 2012].

Postel, Jacques (1981), Genèse de la psychiatrie. Paris, Le Sycomore.

Quétel, Claude (1987), "Présentation”. En: Joseph Daquin, La philosophie de la folie [1791], reedición, Paris, Frénésie.

Reil, Johann Christian (1803), Rhapsodieen über die Anwendung der psychischen Curmethode auf Geisteszerrüttungen. Halle, Curtsche Buchhandlung [Versión castellana: Reil, Johann Christian. Rapsodias sobre el empleo del método de cura psíquica en los trastornos del espíritu. En: AA.VV. El nacimiento de la psiquiatría. Colección Clásicos de la Psiquiatría. Buenos Aires, Polemos, 2012].

Reil, Johann Christian (1808), "Ueber den Begriff der Medicin und ihre Verzweigungen, besonders in Beziehung auf die Berichtigung der Topik der Psychiaterie". En: Reil, JC; Hoffbauer, J.C. (eds.), Beyträge zur Beförderung einer Kurmethode auf psychischem Wege, Halle, Curt'sche Buchhandlung, pp. 161-279. 
Sémelaigne, René (1888), Phillippe Pinel et son oeuvre au point de vue de la médecine mentale. Thèse de doctorat. Paris, Imprimeries réunies.

Stagnaro, Juan Carlos (2014), “Estudio preliminar: Vicenzo Chiarugi y la asistencia de la Locura en Florencia a fines del siglo XVIII". En: Chiarugi Vincenzo, La Locura, sus géneros y especies. Tratado médico-analítico con cien observaciones. 1 o edición en castellano. Colección Clásicos de la Psiquiatría, Buenos Aires, Polemos, pp. 9-80.

Swain, Gladys (1977), Le sujet de la folie, Naissance de la Psychiatrie. Toulouse, Privat.

Weiner, Dora B. (1999), Comprendre et soigner. Philippe Pinel (1745-1826), la médecine de l'esprit. Paris, Fayard. 